\title{
An Organic Cathode for Aqueous Zn Batteries: Taming a Unique Phase Evolution towards Stable Electrochemical Cycling
}

Dipan Kundu*, Pascal Oberholzer $†$, Christos Glaros $\%$, Assil Bouzid, Elena Tervoort, Alfredo

Pasquarello, and Markus Niederberger

Dr. Dipan Kundu, Pascal Oberholzer, Christos Glaros, Dr. Elena Tervoort, Prof. Dr. Markus Niederberger

Multifunctional Materials

Department of Materials

ETH Zurich

Vladimir Prelog Weg 5

8093 Zurich

Switzerland

Email: dipan.kundu@mat.ethz.ch

Dr. Assil Bouzid, Prof. Dr. Alfredo Pasquarello

Chaire de Simulation à l'Échelle Atomique (CSEA)

École Polytechnique Fédérale de Lausanne (EPFL)

CH-1015 Lausanne, Switzerland

$\dagger$ These authors contributed equally to this work

\section{ABSTRACT}

Aqueous zinc batteries are highly attractive for large-scale storage applications owing to their inherent safety, low-cost, and durability. Yet, their advancement is hindered by a dearth of positive host materials (cathode) due to sluggish diffusion of $\mathrm{Zn}^{2+}$ inside solid inorganic frameworks. Here, we report a novel organic host, tetrachloro-1,4-benzoquinone (also called: p-Chloranil), which due to its inherently soft crystal structure can provide reversible and efficient $\mathrm{Zn}^{2+}$ storage. It delivers a high capacity of $\geq 200 \mathrm{mAh} \mathrm{g}^{-1}$ with a very small voltage polarization of $50 \mathrm{mV}$ in a flat plateau around $1.1 \mathrm{~V}$, which equate to an attractive specific energy of $>200 \mathrm{Wh} \mathrm{kg}^{-1}$ at an unparalleled energy efficiency ( 95\%). As unraveled by density functional theory (DFT) calculations, the molecular columns in $\mathrm{p}$-Chloranil undergo a twisted rotation to accommodate $\mathrm{Zn}^{2+}$, thus restricting the volume change $(-2.7 \%)$ during cycling. In-depth characterizations using operando X-ray 
diffraction, electron microscopy, and impedance analysis reveal a unique phase evolution, driven by a phase transfer mechanism occurring at the boundary of solid and liquid phase, which leads to unrestricted growth of discharged/charged phases. By confining the p-Chloranil inside nanochannels of mesoporous carbon CMK-3, we can tame the phase evolution process, and thus stabilize the electrochemical cycling.

\section{INTRODUCTION}

Industrialization brought tremendous societal changes at an extraordinary speed, but it came at huge costs to our environment, and eventually to the health of all living beings. The scientific consensus is very clear now that we are on the verge of a significant and possibly dramatic climate change, which has raised the call for an environmentally sustainable energy economy based on renewable energy sources like solar and wind ${ }^{1}$ However, these energy resources are not viable without efficient energy storage. Therefore, renewable energy generation coupled with electrochemical energy storage has gained enormous prominence around the world, pushing forth considerable advances in the last decade. Lithium ion batteries (LIBs), which are ubiquitous in portable electronics and electric vehicles due to their high energy and power densities, seem like an obvious choice due to their market readiness and validation. ${ }^{2-4}$ However, factors like constrained resource (low abundance of lithium in Earth's crust, vulnerable geopolitical distribution), high cost, safety concerns, and poor recycling infrastructure limit their applicability in large-scale stationary storage where safety, cost, and durability are more of a concern than weight or size..$^{5-8}$

Among beyond lithium ion energy storage systems, multivalent $\left(\mathrm{Zn}^{2+}, \mathrm{Mg}^{2+}, \mathrm{Al}^{3+}\right)$ ion batteries, which not only offer potential cost benefit but may facilitate superior volumetric

capacities, have attracted substantial interests. ${ }^{9-18}$ Aqueous zinc batteries (AZBs) are particularly 
intriguing owing to zinc's high abundance and nontoxicity. ${ }^{19}$ Most importantly, zinc has low redox potential (-0.76 V against Standard Hydrogen Electrode), and high kinetic overpotential for hydrogen evolution, which renders it stable in water. The issues of poor rechargeability and dendrite formation, typical in alkaline electrolytes, is thwarted by using mildly acidic to nearneutral (pH: 4-6) electrolytes, ${ }^{20}$ resulting in rechargeable AZBs with a kinetic voltage window exceeding $2 \mathrm{~V}$ (Figure S1). However, there are not many positive host materials that can reversibly host $\mathrm{Zn}^{2+}$. The problem stems from small size of $\mathrm{Zn}^{2+}$ (Shannon radii of $0.74 \AA$ is comparable to $\mathrm{Li}^{+}$'s $0.76 \AA$ in 6 fold coordination) and its double valence - thus, high charge density, which imposes greater energy barrier for ion mobility in solids due to strong Coulomb interaction with the surrounding anionic lattice. ${ }^{21}$ Although layered and tunneled inorganic structures with wide ion migration pathways have shown promise, use of nano/submicrometer particles is crucial to circumvent the issue of sluggish solid-state diffusion. ${ }^{22-26}$

Organic molecular solids, which have attracted ample interests for both monovalent $\left(\mathrm{Li}^{+}\right.$, $\mathrm{Na}^{+}$, and $\left.\mathrm{K}^{+}\right)$and divalent $\left(\mathrm{Mg}^{2+}\right)$ cation storage in recent times, offer an interesting alternative to inorganic structures. ${ }^{27-31,16}$ These materials, held together by weak intermolecular van der Waals forces, pose only a modest Coulomb repulsion to the diffusing cations. Furthermore, their malleable and soft lattice may allow molecular reorientation for facile and reversible intercalation of hard divalent cations. This is what we observe for $\mathrm{Zn}^{2+}$ intercalation in tetrachloro-1,4benzoquinone (also known as p-Chloranil, where p: para) from mildly acidic aqueous electrolytes. Upon discharge (reduction), p-Chloranil delivers a large specific capacity of $\geq 200 \mathrm{mAh} \mathrm{g}^{-1}$, corresponding to $1 \mathrm{Zn}^{2+} / 2 \mathrm{e}^{-}$reduction per formula unit, in a flat plateau around $1.1 \mathrm{~V}$. Remarkably, structural evolution as probed by Operando X-ray diffraction combined with scanning electron microscopy (SEM), energy dispersive X-ray spectroscopy (EDX), and Electrochemical Impedance 
Spectroscopy (EIS) studies reveal a unique phase transformation between p-Chloranil and Zn-pChloranil (i.e., $\mathrm{Zn}^{2+}$-p-Chloranil ${ }^{2-}$ ) via a water assisted phase transfer mechanism. As a result, the discharged/charged phases grow as large microstructures, a part of which loses contact with the electrode every cycle, leading to capacity fading. Details of the structural change as projected by DFT unravels a rotation molecular columns in p-Chloranil to accommodate the inserting $\mathrm{Zn}^{2+}$, and a relatively small volume change $(-2.7 \%)$ for the $\mathrm{Zn}^{2+}$ storage. To tackle the unhindered evolution of the discharged/charged phase, $\mathrm{p}$-Chloranil was confined inside mesoporous nanochannels of CMK-3 carbon, which dramatically restricts the growth of discharge/charge products, and thus enhances the electrochemical reachargeability and cyclability significantly.

\section{RESULTS AND DISCUSSION}

Electrochemical $\mathbf{Z n}^{2+}$ storage in $\mathbf{p}$-Chloranil. Quinones have been extensively explored as cathode materials for nonaqueous lithium and sodium ion batteries as the carbonyl reduction potential is easy to tune by modifying the functionalization of quinone derivatives. ${ }^{27-29,35}$ While such quinone-based batteries deliver high capacity and energy density, solubility of quinones and corresponding reduction products in typical organic electrolytes and consequent capacity fading with battery cycling pose a serious problem. Interestingly, some quinones like p-Chloranil are completely stable and insoluble in aqueous electrolyte, ${ }^{32}$ thus making it possible to use $\mathrm{p}$-Chloranil as a solid cathode in aqueous batteries. Figure 1a shows the cyclic voltammograms (CV) of pChloranil in $1 \mathrm{M}$ zinc trifluoromethanesulfonate $\left(\mathrm{Zn}(\mathrm{OTf})_{2}\right)$ in water $(\mathrm{pH} \sim 4.45)$. A mixture of $\mathrm{p}$ Chloranil with Super $\mathrm{P}^{\circledR}$ carbon and a water based composite binder (carboxymethylcellulose (CMC) and styrene-butadiene rubber (SBR)) in 60:35:5 weight ratio (see methods in supplementary information for details) served as the positive electrode, while a $\mathrm{Zn}$ foil was used as the negative. Notably, both negative (Zn) and positive (p-Chloranil) redox couples fall well 
within the working potential window of the electrolyte $(\sim 2.5 \mathrm{~V})$ bounded by $\mathrm{H}_{2}$ and $\mathrm{O}_{2}$ evolution reactions (Figure S1a and b). The CV curves are reproducible (Figure S1c) and feature only one cathodic (reduction) and one anodic (oxidation) peak indicating a single step reduction/oxidation of p-Chloranil. Overall, the $\mathrm{Zn}$ - p-Chloranil couple displays an average working voltage of $1.1 \mathrm{~V}$ with a very small hysteresis. The redox kinetics was probed by performing CV at various scan rates as shown in Figure 1b. Increasing scan rate shifts the cathodic peak to lower potential and
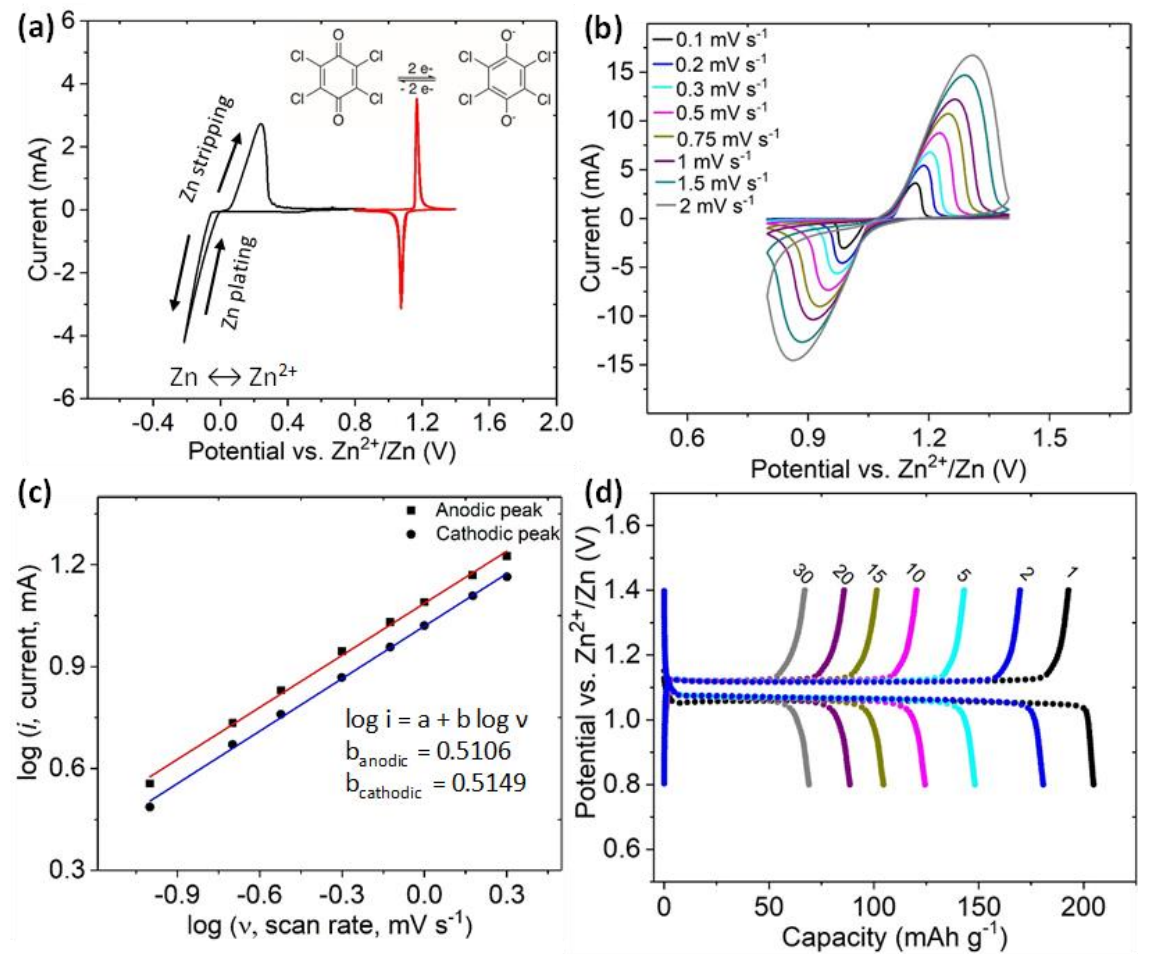

Figure 1. (a) Cyclic voltammograms (CV) of the $\mathrm{Zn}$ anode (black line) against the p-Chloranil cathode (red line) in $1 \mathrm{M} \mathrm{Zn}(\mathrm{OTf})_{2}-\mathrm{H}_{2} \mathrm{O}$ electrolyte at 5 and $0.1 \mathrm{mV} \mathrm{s}^{-1}$, respectively. (b) $\mathrm{CV}$ curves of the p-Chloranil cathode at various scan rates. (c) The logarithmic relationship of peak current with scan rate for the CV curves in (b). (d) Voltage-capacity profiles of the p-Chloranil cathode at a C/5 rate (1C: $217 \mathrm{~mA} \mathrm{~g}^{-1}$ ) in a 0.8-1.4 $\mathrm{V}$ window against $\mathrm{Zn}$. The labels indicate the cycle number.

the anodic peak to higher potential due to worsened polarization. The linear fit of the logarithmic relationship of peak current and scan rate (Figure 1c) reveals that slopes for both anodic and cathodic peaks are nearly 0.5 , suggesting a predominantly diffusion controlled kinetics for faradic 
$\mathrm{Zn}^{2+}$ storage in $\mathrm{p}$-Chloranil. ${ }^{33}$ Presumably, the soft organic lattice combined with facile adsorption of $\mathrm{Zn}^{2+}$ by quinol groups (resulting from quinone reduction) leads to a fast solid-state diffusion of $\mathrm{Zn}^{2+}$. This is further evident from the galvanostatic charge/discharge curves (Figure 1d), which displays a very small polarization of $\sim 50 \mathrm{mV}$ between charge and discharge plateau. The low voltage polarization translates to a very high energy efficiency of $\sim 95 \%$ that is unparallel among state of the art inorganic $\mathrm{Zn}^{2+}$ host materials. The first discharge capacity of $205 \mathrm{mAh} \mathrm{g}^{-1}$ is comparable to $1 \mathrm{Zn}^{2+} / 2 \mathrm{e}^{-}$reaction $\left(217 \mathrm{mAh} \mathrm{g}^{-1}\right)$ occurring in a single plateau, which points to a biphasic electrochemical process. This single plateau feature is quite distinct compared to the nonaqueous $\mathrm{Na}^{+}$(de)insertion in $\mathrm{p}$-Chloranil, where two voltage plateaus appear (separated by 0.3 V) with the formation a stable $\mathrm{Na}_{1.5}$ intermediate phase. ${ }^{34,35}$ Stabilization of the intermediate phase leads to incomplete electrochemical reaction and lower $\mathrm{Na}^{+}$storage capacity of $\sim 150 \mathrm{mAh} \mathrm{g}^{-1}$. Such difference in behavior for $\mathrm{Zn}^{2+}$ vis-à-vis $\mathrm{Na}^{+}$insertion is quite reasonable considering $\mathrm{Zn}^{2+}$ is quite small ( $0.6 \AA$ in 4-fold coordination) compared to $\mathrm{Na}^{+}(1.00 \AA)$, and only one $\mathrm{Zn}^{2+}$ is (de)inserted during the electrochemical process against two $\mathrm{Na}^{+}$. However, dissimilar electrochemical mechanism - specifically, different charge transfer process - in the two systems can also be responsible for the different electrochemical behavior. As evident from Fig. 1d, galvanostatic charging also proceeds in a single plateau, but a fraction of the discharge capacity is not recovered at the end of charge. Incomplete charging in every cycle results in steady capacity decay, leading to a retention of only $70 \mathrm{mAh} \mathrm{g}^{-1}$ at the end of 30 cycles.

Mechanism. Instability and dissolution of p-Chloranil in the electrolyte can be a possible source of the capacity decay. However, that hypothesis could be discarded as visually no coloration of the electrolyte was observed during cell cycling (Figure S2). Cycled electrolyte (after 10 cycles) probed further by FTIR showed no extra band compared to the pure electrolyte, which confirmed 
the absence of dissolution mechanism (Figure S3). In order to understand the influence of phase/structural evolution of $\mathrm{p}$-Chloranil on $\mathrm{Zn}^{2+}$ storage, X-ray diffraction was performed inoperando during electrochemical discharge/charge (Figure 2a and 2b). The commercial pChloranil (commercial) displays a $P 2_{1} / c$ monoclinic crystal structure. Milling with carbon for electrode fabrication leads to small peak broadening, but the structure remain intact (Figure 4).

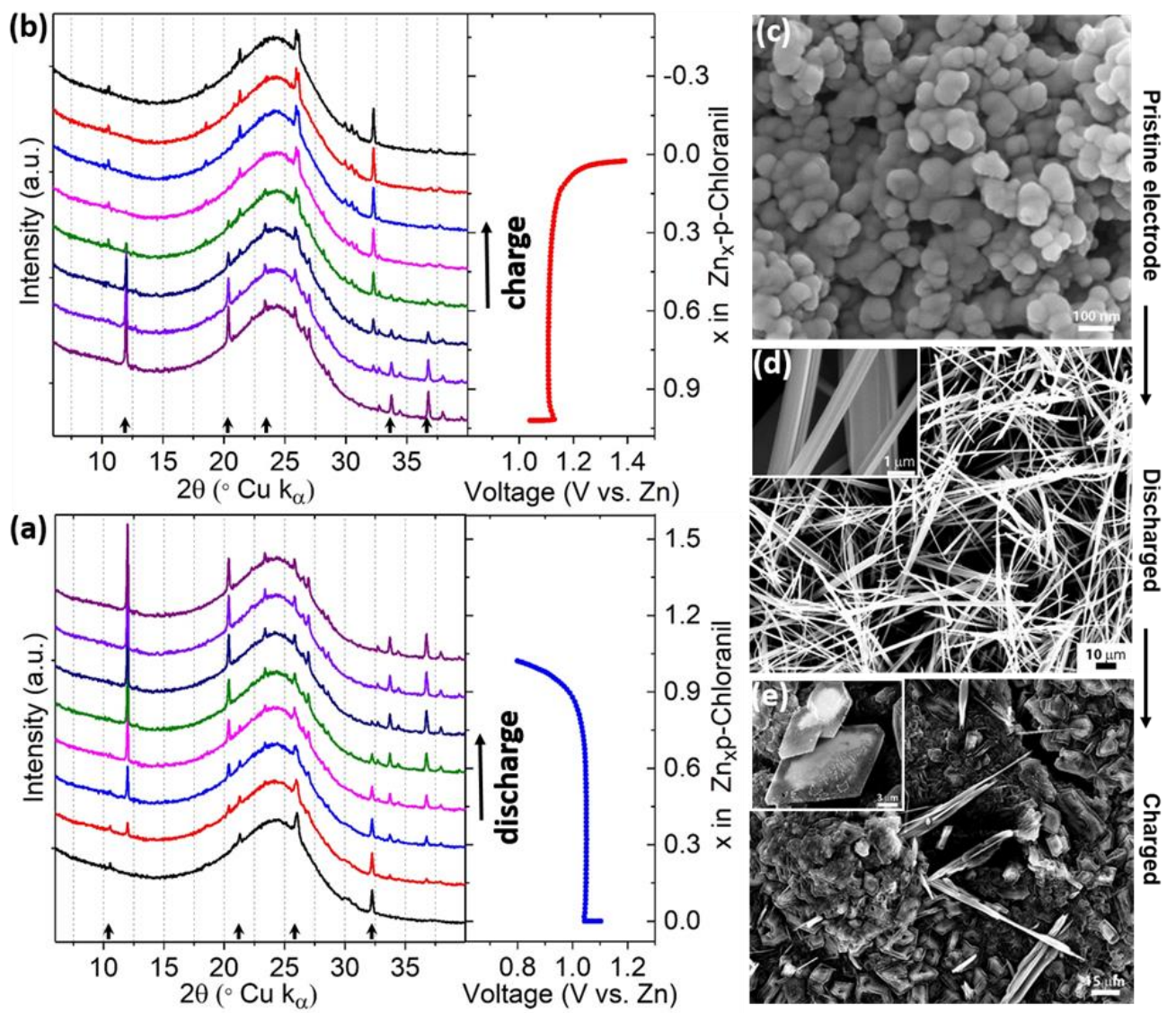

Figure 2. X-ray diffraction patterns of the p-Chloranil electrode during the (a) first discharge (bottom left) (b) and charge (upper left) cycled at a $\mathrm{C} / 5$ rate $\left(1 \mathrm{C}: 217 \mathrm{~mA} \mathrm{~g}^{-1}\right)$ in a $0.8-1.4 \mathrm{~V}$ window against $\mathrm{Zn}$. The black upward facing arrows in the discharge and charge profiles indicate the primary diffraction peaks of pChloranil (charged phase) and Zn-p-Chloranil (discharged phase), respectively. Typical SEM image of the (c) pristine, (d) discharged, and (e) charged p-Chloranil-Super P electrodes showing the morphology evolution as a function of discharge/charge. Inset in (d) and (e) shows the magnified image of the discharged Zn-p-Chloranil fibers and charged p-Chloranil rhombus particles, respectively. 
During discharge, the most intriguing structural transformation is the disappearance of $\mathrm{p}$-Chloranil peaks and the appearance of a completely new set of peaks. With progressive $\mathrm{Zn}^{2+}$ insertion, the intensity of p-Chloranil peaks gradually diminish and the new peaks increase in intensity, although, they all remain unaltered in position. This behavior is indicative of a biphasic structural evolution, which is also apparent from sharp reduction/oxidation peaks in $\mathrm{CV}$ and flat plateau in galvanostatic profile. A reverse evolution occurs during charge with the p-Chloranil peaks being fully restored at the end of the charge process, indicating a high reversibility of the $\mathrm{Zn}^{2+}(\mathrm{de})$ insertion mediated phase transformation. Although a small discrepancy between the inserted and extracted $\mathrm{Zn}^{2+}$ amount (x; Fig. 2a and b) is apparent, no peaks corresponding to the zinc-inserted phase is detected after charge, possibly due to its small fraction $(\sim 2.5 \%)$. Therefore, SEM coupled with EDX was performed to elucidate the composition and nature of the discharged and charged products. SEM revealed a remarkable morphology transformation accompanying the phase transition during discharge/charge process (Figure 2c-e). The pristine electrode consists of carbon particles coated with a film of p-Chloranil. Upon discharge, this film converts to highly homogeneous fibers of several tens of micrometers in length and $\leq 1 \mu \mathrm{m}$ in diameter. Interestingly, on charging, the fibers do not convert back to the film, but transform to nonuniform rhombus particles of 5-20 $\mu \mathrm{m}$ in dimension. In agreement with incomplete electrochemical charging, a small amount of partially reacted fibers is found on the charged electrode. Such morphology evolution during electrochemical cycling can be explained by a water assisted phase transfer mechanism as depicted in Figure 3. A single step $2 \mathrm{e}^{-}$reduction of p-Chloranil during discharge leads to the formation of quinoxide dianions, which react with water protons as a Brønsted base to form soluble quinols at the solid-liquid phase boundary of the cathode and electrolyte. The quinol molecules then instantaneously undergo a metathesis with $\mathrm{Zn}^{2+}$, which are in high abundance in the electrolyte, 
driven by the free energy of formation of solid crystalline $\mathrm{Zn}$-p-Chloranil $\left(\mathrm{ZnC}_{6} \mathrm{Cl}_{4} \mathrm{O}_{2}\right)$. Charge follows a reverse course: water protons react with the surface of the $\mathrm{Zn}$-p-Chloranil fibers to form quinols, releasing $\mathrm{Zn}^{2+}$ into the electrolyte. Soluble quinols are then oxidized on the carbon surface with concomitant crystallization of p-Chloranil. The proposed phase transfer mechanism, which is

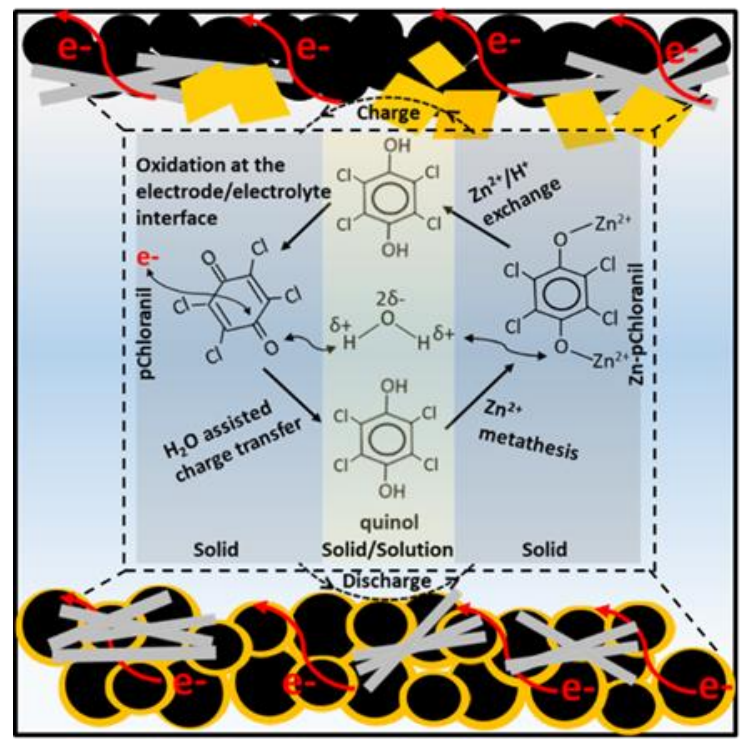

Figure 3. Schematic illustration of the $\mathrm{H}_{2} \mathrm{O}$ assisted phase transfer mechanism for the formation of the discharged - Zn-p-Chloranil (grey fibers) and charged - p-Chloranil (yellow rhombus). Black spherical/oval particles are Super P carbon while the thin yellow shell on the carbon particles (upper section) stands for the $\mathrm{p}$-Chloranil in the pristine electrode. The reactions presented inside the dotted box proceed at the phase boundary of solid electrode and liquid electrolyte. During discharge, p-Chloranil reduces at the solid electrode and reacts with water protons to form quinols at the solid/solution interface. The quinols subsequently undergo metathesis with $\mathrm{Zn}^{2+}$ to form insoluble $\mathrm{Zn}$-p-Chloranil that nucleates and grows as fibers. Charge follows a reverse proceeding: Zn-p-Chloranil exchanges protons with water to form soluble quinols, which then oxidize at the carbon surface and grow as rhombus particles of p-Chloranil.

a well-established technique in organic synthesis, ${ }^{36}$ is believed to proceed at the boundary between immiscible phases (here, solid and liquid), and therefore bulk diffusion of active species to the counter electrode can be neglected. Growth of Zn-p-Chloranil fibers and p-Chloranil rhombus particles most occur anisotropically, promoted most likely by the surface energy anisotropy of different crystal facets. Careful EDX analysis of the discharged - fibers, charged - rhombus 
particle and the remnant fibers in the charged electrode confirmed their composition. The fibers are indeed the result of $1 \mathrm{Zn}^{2+}$ reaction per p-Chloranil unit, in perfect accordance with the electrochemical results, while the rhombus crystals are recharged p-Chloranil, containing a negligible amount of zinc (Figure S5).

EIS measurements were performed at different state of discharge/charge (as indicated in Figure 4a) to understand the kinetics of the p-Chloranil - Zn-p-Chloranil phase transformation closely. The corresponding Nyquist plots are shown in Figure $\mathbf{4 b}$, where the frequency decreases from left to right on the ' $x$ ' $(\operatorname{Re}(Z))$ axis. All the impedance profiles can be decomposed into the following components: a small Ohmic resistance at high frequencies ( $\geq 200 \mathrm{kHz})$ corresponding to the electrolyte and contact resistance in the cell; a high-medium frequency $(200 \mathrm{kHz}-100 \mathrm{~Hz})$ capacitive semicircle related to the electrochemical charge transfer at the interface; a medium-low frequency $(100 \mathrm{~Hz}-1 \mathrm{~Hz})$ inductance loop typically associated with the relaxation of species (of ions, molecules) adsorbed on the electrode surface $;{ }^{37}$ and a low frequency $(<1 \mathrm{~Hz})$ solid-state diffusion component. EIS measurements performed under different dc bias current further validated the assignment of the capacitive semicircle to the charge transfer step (Figure S6). A clear shrinkage of the capacitive arc is observed with increasing bias current as anticipated for a charge transfer process. ${ }^{38}$ Overall, the charge transfer resistance during charge becomes half that of discharge, indicating a slightly faster kinetics for the charge reaction. This is also evident from the smaller $(9 \mathrm{mV})$ overpotential (difference between the charge/discharge potential and equilibrium potential at point $\mathrm{B} / \mathrm{D})$ during charge compared to discharge $(20 \mathrm{mV})$. This can be explained by the relatively large surface area of Zn-p-Chloranil microfibers accessible for the phase transfer mediated charge transfer process (see above). Intriguingly, on a closer look the capacitive semicircle appears like a combination of two depressed capacitive arc of slightly 

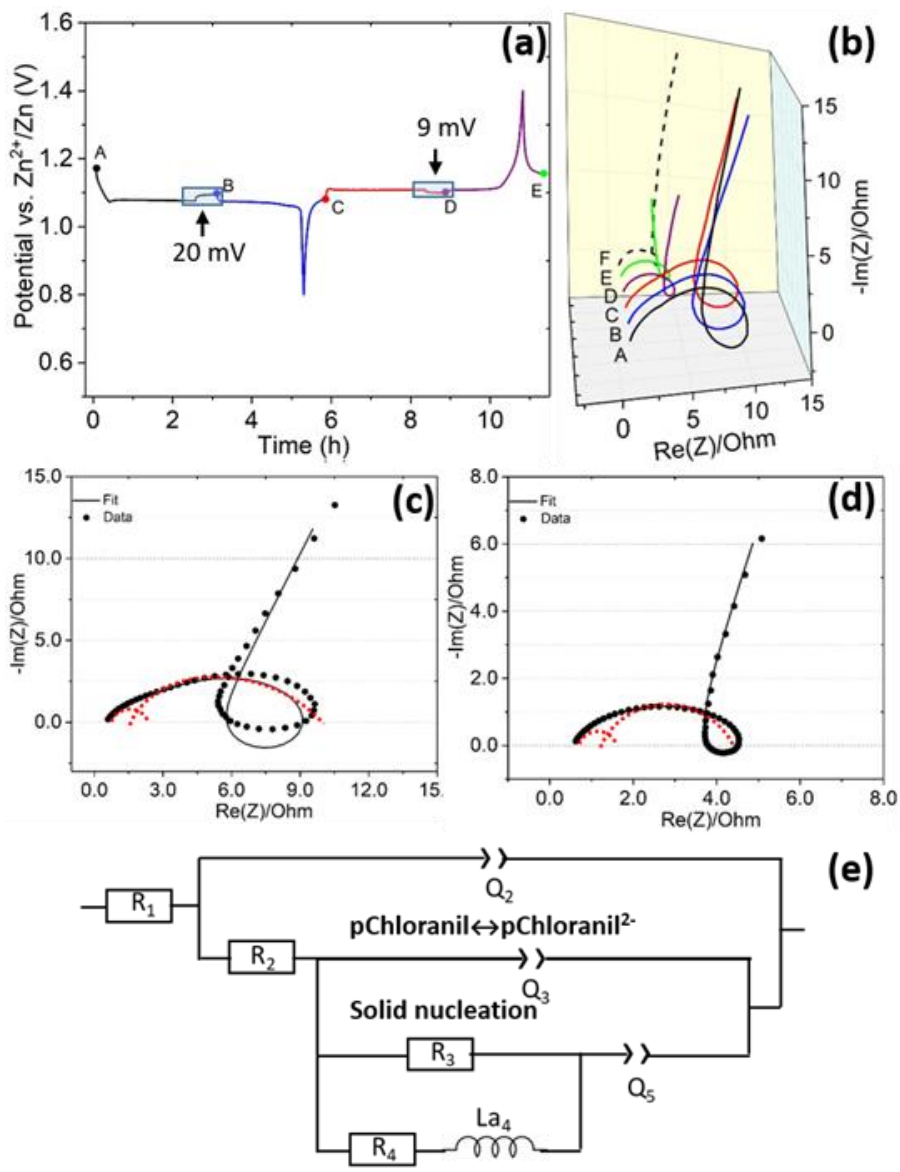

(e)

Figure 4. (a) Galvanostatic discharge/charge profiles of the p-Chloranil cathode where the labels A to E indicate different depth of discharge/charge at which impedance (EIS) measurements were conducted. The cells were equilibrated for $0.5 \mathrm{~h}$ before each measurement. The blue boxes at point $\mathrm{B}$ and $\mathrm{D}$ highlight the voltage polarization during discharge and charge, respectively, as obtained from the difference of discharge/charge voltage and the equilibrium voltage. (b) Nyquist plots corresponding to the impedance measurements. Fitting (solid black line) of the impedance data (black circles) collected at point (c) B and (d) D with the model shown in (e). The red doted arcs are for guidance; the first one (left, high frequency) corresponds to the ion transfer at the interface, while the second one (right, low frequency) represents the nucleation of solid Zn-p-Chloranil on the cathode. R, Ohmic resistance; Q, non-ideal capacitor; La, nonideal inductor.

different time constants. This became apparent while fitting the impedance data with a model circuit, as a single capacitive arc lead to inferior fit and odd parameter values. Figure $\mathbf{4 c}$ and $4 \mathbf{d}$ show the fitting of the Nyquist impedance collected at point B and D, which are around halfway during discharge and charge, respectively. Better fit was obtained considering a two-step charge 
transfer model (Figure 4e) for the transformation of p-Chloranil to Zn-p-Chloranil: a fast ion $\left(\mathrm{Zn}^{2+}\right)$ transfer (higher frequency capacitive arc) at the electrolyte/electrode interface (for $\mathrm{p}$ Chloranil to $\mathrm{p}$-Chloranil ${ }^{2-}$ transformation), followed by a slightly slower (lower frequency capacitive arc) nucleation of the solid Zn-p-Chloranil on the cathode surface. The two arcs are manually denoted in Fig. 4c and 4d (red dotted arcs) for guidance. As expected, the resistance to ion transfer remains nearly the same from discharge to charge; however, the resistance associated with the solid nucleation (or dissolution during charge) decreases by more than half during charge (Table S1). This is an outcome of the large exposed surface area of the Zn-p-Chloranil fibers available for the charge transfer reaction, as discussed above.

DFT insight into the structural transformation. In order to gain further insight into the $\mathrm{Zn}^{2+}$ insertion process, it was imperative to obtain structural information of the resultant phase, but quality of the diffraction data forbade an ab initio structure solution. Therefore, first principles density functional calculations were carried out to elucidate the possible structure of $\mathrm{Zn}-\mathrm{p}$ Chloranil, i.e., $\mathrm{Zn} \cdot \mathrm{C}_{6} \mathrm{Cl}_{4} \mathrm{O}_{2}$ (see experimental methods for details). Table 1 lists the experimentally obtained lattice parameters, compared with the values predicted by DFT for the lowest energy solution. While experimental data is indexed to a monoclinic cell with a $\beta$ angle of $94.03^{\circ}$, the DFT structure shows a slightly distorted monoclinic phase with $\beta=99.58^{\circ}$. Overall, the predicted DFT model shows a good agreement with the experimental results and reproduces the primary diffraction peaks of the experimental pattern within shifts (Figure S7). It is important to note that the experimental pattern was obtained from the operando cell, and due to the anisotropic structure of the discharged Zn-p-Chloranil, many diffraction peaks are low intensity or missing. Figure 5a and $5 \mathrm{~b}$ show the fully relaxed structure of pure $\mathrm{p}$-Chloranil and $\mathrm{Zn}^{2+}$ inserted $\mathrm{p}$-Chloranil, respectively. Remarkably, upon insertion of $\mathrm{Zn}^{2+}$ the $\mathrm{p}$-Chloranil molecules undergo a squeezing 


\begin{tabular}{|c|c|c|}
\hline Cell parameters & Experiment & DFT \\
\hline$a(\AA)$ & 7.37 & 7.37 \\
\hline$b(\AA)$ & 4.64 & 4.62 \\
\hline$c(\AA)$ & 12.65 & 12.51 \\
\hline$\alpha\left(^{\circ}\right)$ & 90 & 88.90 \\
\hline$\beta\left(^{\circ}\right)$ & 94.03 & 99.58 \\
\hline$\gamma\left({ }^{\circ}\right)$ & 90 & 90.19 \\
\hline
\end{tabular}

Table 1. Comparison of the cell parameters of Zn-p-Chloranil obtained by indexing the experimental X-ray powder pattern, and DFT calculations.

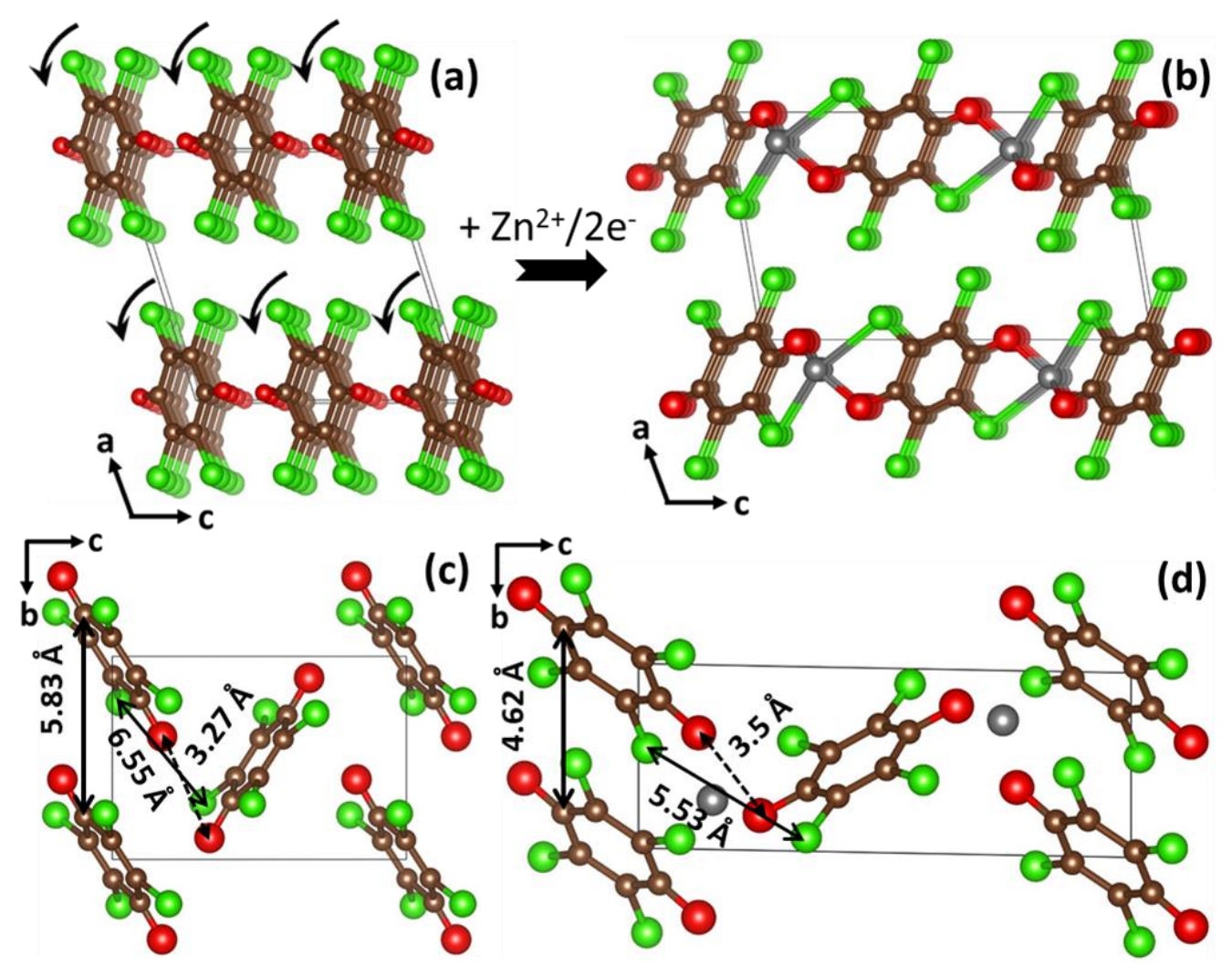

Figure 5. Structural models of (a) p-Chloranil and (b) $\mathrm{Zn}$-p-Chloranil $\left(\mathrm{Zn}^{\circ} \mathrm{C}_{6} \mathrm{Cl}_{4} \mathrm{O}_{2}\right)$ as obtained from DFT structural optimization. The curved arrows in (a) show the direction of the rotation of the p-Chloranil molecular columns upon $\mathrm{Zn}^{2+}$ insertion. Color code: $\mathrm{C}$ (brown), $\mathrm{Cl}$ (green), $\mathrm{O}$ (red), and $\mathrm{Zn}$ (grey). $\mathrm{Cl}-\mathrm{Cl}$, $\mathrm{O}-\mathrm{O}$, and $\mathrm{C}-\mathrm{C}$ (which is actually the distance between two adjacent aromatic rings in the molecular column) distances in (c) p-Chloranil and (d) Zn-p-Chloranil.

reorientation in order to accommodate the inserted ions. The reorientation can be understood as the rotation of stacked Chloranil columns driven by the insertion of $\mathrm{Zn}^{2+}$ ions, which increases the O-O distance and decreases the $\mathrm{Cl}-\mathrm{Cl}$ distance compared to Chloranil (Figure 5c and 5d). The atomic distances change as a consequence of the coordination of $\mathrm{Zn}^{2+}$ with two $\mathrm{O}$ and $\mathrm{Cl}$ of two 
Chloranil molecules of adjacent columns, housing $\mathrm{Zn}^{2+}$ in a slightly distorted tetrahedral environment. Moreover, $\mathrm{Zn}^{2+}$ accommodation results in shortening of the $\mathrm{d}$ spacing between (100) and (010) planes (Figure S8) as a result of improved packing of the organic structure, which is also evident from the small contraction $(-2.7 \%)$ of the unit cell volume from $432 \AA^{3}$ to $420 \AA^{3}$. Compact structure also stems from the aromatization of the carbon ring upon discharge, which leads to an enhanced $\pi-\pi$ stacking ${ }^{39}$ in the Chloranil column along $\boldsymbol{b}$, decreasing the intermolecular distance from $5.83 \AA$ in Chloranil to $4.62 \AA$ in Zn-Chloranil (Figs 5c and d). Interestingly, a rotation of the Chloranil stacks has also been predicted by DFT calculation for $\mathrm{Na}^{+}$insertion. ${ }^{[34]}$ However, in a sharp contrast to the zinc system, one $\mathrm{Na}^{+}$insertion is expected to cause $8 \%$ volume increase, which doubles up for $2 \mathrm{Na}^{+}$insertion, due to large size of $\mathrm{Na}^{+}$(and its higher coordination number) and strong electrostatic repulsion between Na-ions.

p-Chloranil confinement in CMK-3: improved electrochemical cycling. With the knowledge of the discharge/charge mechanism and accompanying morphology evolution, it was clear that the uncontrolled evolution of the discharged/charged phase must be contained to enable full rechargeability and thus better cyclability in this system. Taking a cue from the work by Nazar group on Li-S battery, where they confined the sulfur inside mesoporous nanochannels of CMK3 carbon to mitigate the polysulfide shuttle, ${ }^{40}$ we used CMK-3 as the conductive host for pChloranil. As-synthesized CMK-3 shows a large surface area of $736 \mathrm{~m}^{2} \mathrm{gm}^{-1}$ and pore (diameter $3.5 \mathrm{~nm}$ ) volume of $0.65 \mathrm{~cm}^{3} \mathrm{~g}^{-1}$ (Figure S9), which was impregnated with p-Chloranil from a solution in acetone. After p-Chloranil imbibition, the surface area decreases by more than 15 times to $40 \mathrm{~m}^{2} \mathrm{~g}^{-1}$ and total pore volume decreases to $0.08 \mathrm{~cm}^{3} \mathrm{~g}^{-1}$, indicating to partial filling of the mesopores. Filling of the CMK-3 mesopores is more obvious from the pore size distribution plot (Figure S10), which shows that the pore volume corresponding to the nanochannels (3 - $5 \mathrm{~nm}$ 
diameter pores) decreases markedly upon p-Chloranil infusion. It is also clear that some volume remain unfilled, which along with the residual micropores allow percolation of the electrolyte inside the composite structure.

For the electrode fabrication, the ratio of p-Chloranil to carbon (in the form of CMK-3-pChloranil composite; no Super P was added) and binder was kept the same (i.e., 60:35:5 by weight) as in the Super P electrode. As evident from Figs 6a and $\mathbf{6 b}$ the CMK-3-p-Chloranil composite (60:35) shows much improved electrochemical cyclability relative to the Super P electrode. An initial capacity of $\sim 140 \mathrm{mAh} \mathrm{g}^{-1}$ is delivered at $0.1 \mathrm{C}\left(\mathrm{C} / 10 ; 1 \mathrm{C}: 217 \mathrm{~mA} \mathrm{~g}^{-1}\right)$ rate in the initial cycle, which then stabilizes at 100-110 $\mathrm{mAh} \mathrm{g}^{-1}$ after the first three cycles. Increasing the current to $0.2 \mathrm{C}(\mathrm{C} / 5)$ reduces the starting capacity to $125 \mathrm{mAh} \mathrm{g}^{-1}$, but cycling stability remains unaffected. The capacity fading observed in the initial cycles can be ascribed to the p-Chloranil that remains on the surface of the mesoporous carbon. Note that poor conductivity $\left(0.2 \mathrm{~S} \mathrm{~cm}^{-1}\right)$ of CMK-3 increases the overall impedance of the electrode (Figure S11) leading to a higher charge/discharge polarization (Figure S12) and lower specific capacity compared to the Super P electrode. The origin of the improved cycling becomes more apparent after SEM inspection of the discharged and charged electrodes (Figure 6c-e). Even though CMK-3 does not completely contain the growth of the Zn-p-Chloranil within its pores, in a remarkable contrast to the Super P electrode Zn-pChloranil grows as $\leq 50 \mathrm{~nm}$ nanoribbons, which appear to originate out of the CMK-3 pores and entangle the CMK-3 particles. Smaller dimension and intimate wiring with carbon (CMK-3) result in superior rechargeability of $\mathrm{Zn}$-p-Chloranil, which in turn improves the cycling behavior significantly. Similarly, charging of Zn-p-Chloranil from CMK-3 leads to a film like morphology, very unlike the large particles that appear on charging of the Super P based electrode. Such film morphology leads to better electrical contact within the electrode, which in turn influences efficient 
active material utilization and renders better electrochemical cyclability. Therefore, it is safe to say that the CMK-3 not only acts as an electronic conduit for the confined p-Chloranil, its mesoporous nanochannels serve as microscopic electrochemical reaction chambers. The confinement induces a complex redox process, restricting the nucleation and growth of discharged and charged products, which plays a vital role in improving the electrochemical cyclability.
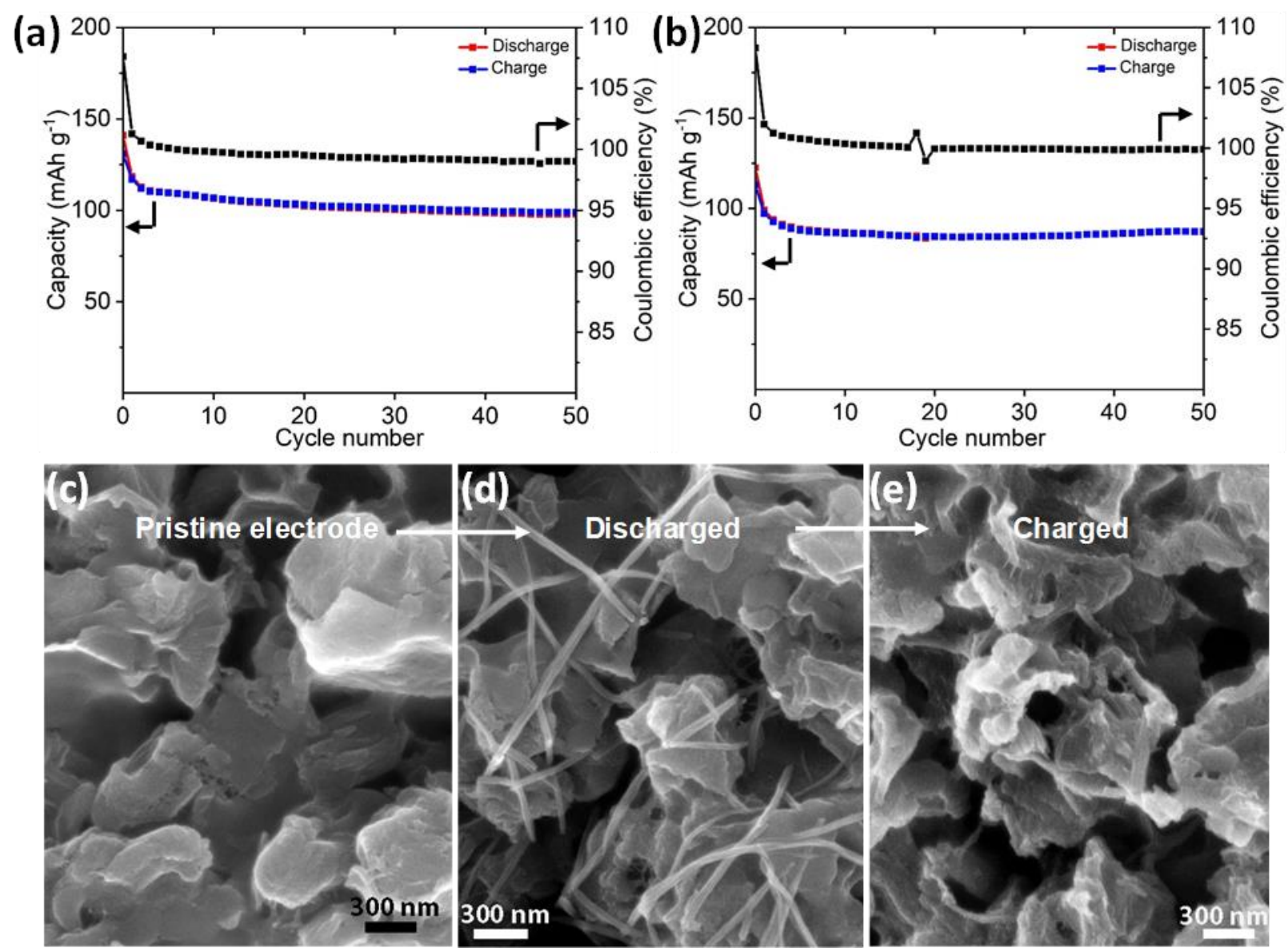

Figure 6. Galvanostatic cyclability and corresponding Coulombic efficiency of the p-Chloranil infused CMK-3 electrode at a C/10 (a) and C/5 rate (b) (1C: $\left.217 \mathrm{~mA} \mathrm{~g}^{-1}\right)$. Representative SEM images of the pristine (c), discharged (d), and charged (e) electrode, showing the formation of the $\mathrm{Zn}$-p-Chloranil nanoribbons upon discharge, which disappears on charging. Here, p-Chloranil in the pristine and the charged electrode has a film morphology. 


\section{CONCLUSION}

In summary, we demonstrate that organic materials with crystal structure pinned by weak van der Waals forces could be ideal for facile $\mathrm{Zn}^{2+}$ storage. Malleable lattice of $\mathrm{p}$-Chloranil allows a unique rotation of stacked molecular columns to make way for incoming $\mathrm{Zn}^{2+}$, and thus minimize the volume change (-2.7\%) accompanying $\mathrm{Zn}^{2+}$ cycling. The structural flexibility facilitates a high specific capacity of $\geq 200 \mathrm{mAh} \mathrm{g}^{-1}$ at $1.1 \mathrm{~V}$ with a very small voltage polarization for $1 \mathrm{Zn}^{2+} / 2 \mathrm{e}^{-}$ reaction. While the specific energy $\left(\sim 200 \mathrm{Wh} \mathrm{kg}^{-1}\right)$ is attractive and on par with state of the art $\mathrm{Zn}^{2+}$ storage materials, the energy efficiency (95\%) is significantly ahead of those. The problem of active material dissolution, typically associated with quinone electrochemistry in nonaqueous batteries, is absent, but an unprecedented phase evolution between p-Chloranil and discharged Znp-Chloranil is central to the electrochemical process here. Water assisted phase transfer mediated discharge and charge reactions lead to an unrestricted growth of large microstructures of the discharged/charged phases, jeopardizing the electrochemical rechargeability. Even though this is the first report on such a process in rechargeable metal-ion batteries, we believe this is not unusual for organic materials whose physical properties change as a function of redox state. Confining pChloranil inside mesoporous nanochannels of CMK-3 ensures intimate contact of the discharged and charged phases with the conductive carbon framework and their restricted growth originating from inside the nanopores, which affords improved rechargeability and excellent cyclability. Thus, we show that the nanoconfinement strategy, which is typically used in sulfur cathode design to entrap soluble polysulfide species in Li-S batteries, is also extendable to rechargeable metal-ion batteries, particularly to tame the complex electrochemical phase evolution. Our strategy paves the way for the departure from conventional cathode design, and may further inspire ingenious 
approaches to spatially contain the growth of discharge/charge phases, which might be the key to cycle other organic host materials as well in aqueous rechargeable batteries.

\section{ASSOCIATED CONTENT}

Supporting information contains experimental methods, additional electrochemical results, impedance analysis results, SEM-EDX analysis of discharged/charged electrodes, nitrogen adsorption-desorption analysis of CMK-3 and p-Chloranil infused CMK-3

\section{ACKNOWLEDGEMENTS}

We thank the Swiss National Science Foundation (SNSF) for the financial support for this work through their Ambizione grant to D. K. We also acknowledge ScopeM (ETH Zurich) for providing the SEM facilities.

\section{REFERENCES}

1. United Nations/Framework Convention on Climate Change, Adoption of the Paris Agreement, 21st Conference of the Parties, Paris: United Nations, 2015.

2. Larcher, D.; Tarascon, J. M. Towards greener and more sustainable batteries for electrical energy storage. Nature Chem. 2015, 7, 19-29.

3. Nayak, P. K.; Yang, L.; Brehm, W.; Adelhelm, P. From lithium-ion to sodium-ion batteries: advantages, challenges, and surprises. Angew. Chem. Int. Ed. 2018, 57, 102-120.

4. Goodenough, J. B.; Park, K-S. The Li-ion rechargeable battery: a perspective. J. Am. Chem. Soc. 2013, 135, 1167-1176.

5. Wanger, C. T. The lithium future - resources, recycling, and the environment. Conservation Lett. 2011, 4, 202-206.

6. Jacoby, M. Safer Lithium-ion Batteries. Safer lithium-ion batteries. Chem. \& Eng. News, 2013, 91, 33-37.

7. Wessells, C. D.; Huggins, R. A.; Cui, Y. Copper hexacyanoferrate battery electrodes with long cycle life and high power. Nat. Commun. 2011, 2, 550.

8. Pasta, M., Wessells, C. D., Liu, N., Nelson, J., McDowell, M. T., Huggins, R. A., Toney, M. F. \& Cui, Y. Full open-framework batteries for stationary energy storage. Nat. Commun. 2014, 5, 3007.

9. Ju, C.; Chen, Y.; Shi, S.; Li, J.; Kang, F.; Su, D.. Secondary batteries with multivalent ions for energy storage. Sci. Rep. 2015, 5. 14120. 
10. Muldoon, J.; Bucur, B. C.; Gregory, T. Quest for nonaqueous multivalent secondary batteries: magnesium and beyond Chem. Rev. 2014, 114, 11683-11720.

11. Alfaruqi, M. H.; Mathew, V.; Song, J.; Kim, S.; Islam, S.; Pham, D. T.; Jo, J.; Kim, S.; Baboo, J. P.; Xiu, J.; Lee, K-S.; Sun, Y-K.; Kim, J. Electrochemical zinc intercalation in lithium vanadium oxide: a high-capacity zinc-ion battery cathode. Chem. Mater. 2017, 29, 1684-1694.

12. Trocoli, R.; Mantia, F. L. An aqueous zinc-ion battery based on copper hexacyanoferrate. ChemSusChem, 2015, 8, 481-485.

13. Sun, X.; Duffort, V.; Mehdi, L. B.; Browning, N. D.; Nazar, L. F. Investigation of the mechanism of $\mathrm{Mg}$ insertion in birnessite in nonaqueous and aqueous rechargeable $\mathrm{Mg}$-ion batteries. Chem. Mater. 2016, 28, 534-542.

14. Canepa, P.; Gautam, G. S.; Hannah, D. C.; Malik, R.; Liu, M.; Gallaghar, K. G.; Persson, K. A.; Ceder, G. Odyssey of multivalent cathode materials: open questions and future challenges. Chem. Rev. 2017, 117, 4287-4341.

15. Liang, Y.; Feng, R.; Yang, S.; Ma, H.; Liang, J.; Chen, J. Rechargeable Mg batteries with graphenelike $\mathrm{MoS}_{2}$ cathode and ultrasmall Mg nanoparticle anode. Adv. Mater. 2011, 23, 640-643.

16. Pan, B.; Huang, J.; Feng, Z.; Zeng, L; He, M.; Zhang, L.; Vaughey, J. T.; Bedzyk, M. J.; Fenter, P.; Zhang, Z.; Burrel, A. K.; Liao, C. Polyanthraquinone-based organic cathode for. high-performance rechargeable magnesium-ion batteries, Adv. Energy Mater. 2016, 6, 1600140.

17. Lin, M. C.; Gong, M.; Lu, B.; Wu, Y.; Wang, D-Y.; Guan, M.; Angell, M.; Chen, C.; Yang, J.; Hwang, B-J.; Dai, H. An ultrafast rechargeable aluminium-ion battery. Nature, 2015, 520, 325-328.

18. Walter, M.; Karvchyk, K. V.; Böfer, C.; Widmer, R; Kovalenko, M. V. Adv. Mater. DOI: 10.1002/adma.201705644, 2018.

19. Zhang, X. G. Corrosion and electrochemistry of zinc, Springer US, (1996).

20. Kundu, D.; Adams, B. D.; Duffort, V.; Vajargah, S. H.; Nazar L. F., Nature Energy, 2016, 1,16119.

21. Rong, Z.; Malik, R.; Canepa, P.; Gautam, G-S.; Liu, M.; Jain, A.; Persson, K.; Ceder, G. Materials design rules for multivalent ion mobility in intercalation structures. Chem. Mater. 2015, 27, 6016-6021.

22. Xu, C. J.; Li, B. H.; Du, H. D.; Kang. F. Y. Energetic zinc ion chemistry: the rechargeable zinc ion battery. Angew. Chem. Int. Ed., 2012, 51, 933-935.

23. Gupta, T. et al. Improving the cycle life of a high-rate, high-potential aqueous dual-ion battery using hyper-dendritic zinc and copper hexacyanoferrates. J. Power Sources, 325, 22-29 (2016).

24. Xia, C.; Guo, J.; Lei, Y.; Liang, H.; Zhao, C.; Alshraeef, H. N. Rechargeable aqueous zinc-ion battery based on porous framework zinc pyrovanadate intercalation cathode. Adv. Mater. 2018, 30, 1705580.

25. Zhang, L. Y.; Chen, L.; Zhou, X. F.; Liu, Z. P. Towards high-voltage aqueous metal-ion batteries beyond 1.5 V: the zinc/zinc hexacyanoferrate system. Adv. Energy Mater. 2015, 5, 1400930. 
26. He, P.; Yan, M.; Zhang, G.; Sun, R.; Chen, L.; An, Q.; Mai, L. et al. Layered VS 2 nanosheet-based aqueous $\mathrm{Zn}$ ion battery cathode. Adv. Energy Mater. 2017, 7, 1601920.

27. Wang, H.; Yuan, S.; Si, Z.; Zhang, X. Multi-ring aromatic carbonyl compounds enabling high capacity and stable performance of sodium-organic batteries. Energy Environ. Sci. 2015, 8, 31603165 .

28. Wang, S.; Wang, L.; Zhang, K.; Zhu, Z.; Tao, Z.; Chen, J. Organic $\mathrm{Li}_{4} \mathrm{C}_{8} \mathrm{H}_{2} \mathrm{O}_{6}$ Nanosheets for lithium-ion batteries. Nano Lett. 2013, 13, 4404-4409.

29. Wang, S.; Wang, L.; Zhu, Z.; Hu, Z.; Zhao, Q.; Chen, J. All organic sodium-ion batteries with $\mathrm{Na}_{4} \mathrm{C}_{8} \mathrm{H}_{2} \mathrm{O}_{6}$, Angew. Chem., Int. Ed. 2014, 53, 5892-5896.

30. Fang, C.; Huang, Y.; Zhang, W.; Han, J.; Deng, Z.; Cao, Y.; Yang, H. Routes to high energy cathodes of sodium-ion batteries, Adv. Energy Mater. 2016, 6, 1501727.

31. Perez, I. A. R.; Yuan, Y.; Bommier, C.; Wang, X.; Ma, L.; Leonard, D. P.; Lerner, M. M.; Carter, R. G.; Wu, T.; Greaney, P. A.; Lu, J.; Ji, X. Mg-ion battery electrode: an organic solid's herringbone structure squeezed upon Mg-ion insertion, J. Am. Chem, Soc. 2017, 139, 13031-13037.

32. Alt, H.; Binder, H.; Klempert, G.; Köhling, A.; Sandstede, G. Evaluation of organic battery electrodes: voltammetric study of the redox behavior of solid quinones. J. App. Electrochem. 1972, 2, 193-200.

33. Brezesinski, T.; Wang, J.; Polleux, J.; Dunn, B.; Tollbert, S. H. Templated nanocrystal-based porous $\mathrm{TiO}_{2}$ films for next-generation electrochemical capacitors. J. Am. Chem. Soc. 2009, 131, 1802-1809.

34. Araujo, R. B.; Banerjee, A.; Ahuja, R. Divulging the hidden capacity and sodiation kinetics of $\mathrm{Na}_{\mathrm{x}} \mathrm{C}_{6} \mathrm{Cl}_{4} \mathrm{O}_{2}$ : a high voltage organic cathode for sodium rechargeable batteries. J. Phys. Chem. C, 2017, 121, 14027-14036.

35. Kim, H.; Kwon, J. E.; Lee, B.; Hong, J.; Lee, M.; Park, S. Y.; Kang, K. High energy organic cathode for sodium rechargeable batteries. Chem. Mater. 2015, 27, 7258-7264.

36. Fita, P. Toward understanding the mechanism of phase transfer catalysis with surface second harmonic generation. J. Phys. Chem. C, 2014, 118, 23147-23153.

37. Cachet, C. et al. EIS investigation of zinc dissolution in aerated sulphate medium. Part II: zinc coatings. Electrochim. Acta, 2002, 47, 3409-3422.

38. Juang, L. W.; Kollmeyer, P. J.; Jahns, T. M.; Lorenz, R. D. Improved nonlinear model for electrode voltage-current relationship for more consistent online battery system identification. IEEE Trans. Ind. Appl. 2013, 49, 1480-1488.

39. Piovesan, D.; Minervini, G.; Tosattoo, S. C. E. The RING 2.0 web server for high quality residue interaction networks. Nucleic Acids Res, 2016, 44, W367-W374. 
40. Ji, X.; Lee, K. T.; Nazar, L. F. A highly ordered nanostructured carbon-sulphur cathode for lithiumsulphur batteries. Nature Mater. 2009, 8, 500-506.

\section{ABSTRACT GRAPHIC (TOC Figure)}

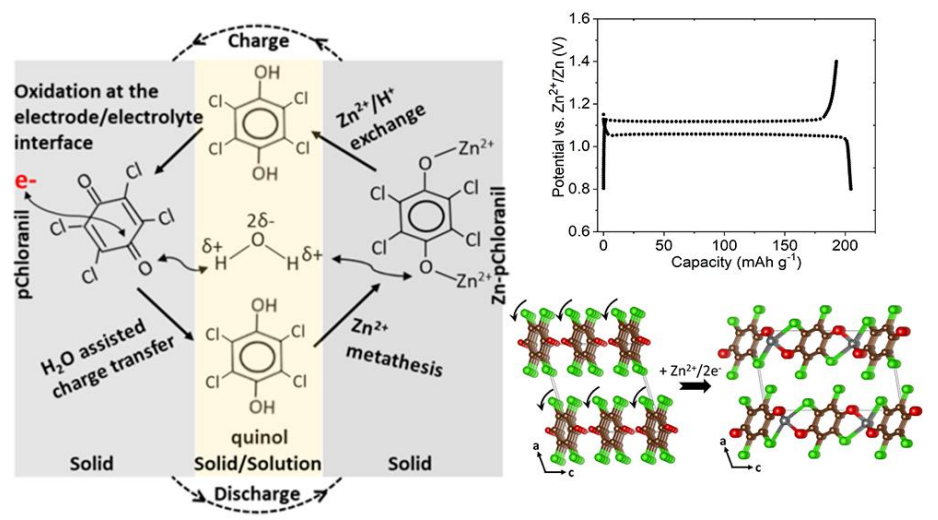

